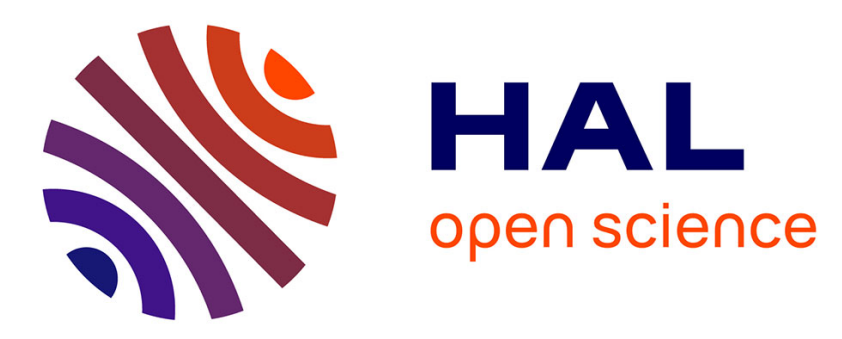

\title{
Devs modelling and simulation for healthcare process application for hospital emergency department
}

Mariem Sbayou, Youssef Bouanan, Grégory Zacharewicz, Judicaël Ribault, Julien Francois

\section{- To cite this version:}

Mariem Sbayou, Youssef Bouanan, Grégory Zacharewicz, Judicaël Ribault, Julien Francois. Devs modelling and simulation for healthcare process application for hospital emergency department. Proceeding ANSS '17 Proceedings of the 50th Annual Simulation Symposium, Apr 2017, Virginia Beach, United States. hal-01934212

\section{HAL Id: hal-01934212 \\ https://hal.science/hal-01934212}

Submitted on 1 Feb 2021

HAL is a multi-disciplinary open access archive for the deposit and dissemination of scientific research documents, whether they are published or not. The documents may come from teaching and research institutions in France or abroad, or from public or private research centers.
L'archive ouverte pluridisciplinaire HAL, est destinée au dépôt et à la diffusion de documents scientifiques de niveau recherche, publiés ou non, émanant des établissements d'enseignement et de recherche français ou étrangers, des laboratoires publics ou privés. 


\title{
DEVS MODELLING AND SIMULATION FOR HEALTHCARE PROCESS APPLICATION FOR HOSPITAL EMERGENCY DEPARTMENT
}

\author{
Mariem Sbayou \\ IMS Laboratory, UMR 5218 \\ University of Bordeaux \\ 351, Cours de la Libération \\ Talence, 33400, FRANCE \\ mariem.sbayou@u-bordeaux.fr
}

\author{
Youssef Bouanan \\ GREThA Laboratory, UMR CNRS 5113 \\ University of Bordeaux \\ Avenue Léon Duguit \\ Pessac, 33600, FRANCE \\ youssef.bouanan@u-bordeaux.fr
}

\author{
Gregory Zacharewicz \\ IMS Laboratory, UMR 5218 \\ University of Bordeaux \\ 351, Cours de la Libération \\ Talence, 33400, France
}

\author{
Judicael Ribault \\ IMS Laboratory, UMR 5218 \\ University of Bordeaux \\ 351, Cours de la Libération \\ Talence, 33400, France \\ judicael.ribault@u-bordeaux.fr
}

\author{
Julien François \\ IMS Laboratory, UMR 5218 \\ University of Bordeaux \\ 351, Cours de la Libération \\ Talence, 33400, France \\ julien.francois@u-bordeaux.fr
}

\begin{abstract}
Healthcare organizations are facing huge daily challenges which led them trying to give the best solutions in order to manage their resources and deliver a best quality of services. The recent adoption of Business Process Management (BPM) in healthcare organizations is dealing with the development of performance indicators in this domain to help healthcare providers structuring the interaction of information between systems and people. Moreover, there are a lot of available methods and tools for BPM that afford different manners to simulate models. Nevertheless in these process models, the resource handling is frequently missing or it is defined in a simplistic way. In this paper, we present the application of BPM in healthcare sector, using the Business Process Model and Notation (BPMN), coupled with a multidimensional Agent Based Model (ABM) of multidimensional organizational network of resources and geographical positioned population on a territory.
\end{abstract}

Keywords: Healthcare organizations, quality services, Workflow, Distributed Simulation, performance.

\section{INTRODUCTION}

Healthcare system is known as a set of organizations, institutions, resources and people whose primary goal is to improve healthcare service quality. This system has four components: request of care, offer of care, funding and piloting. Healthcare environment is characterized as a very dynamic work environment, in which clinicians rapidly switch between work activities and tasks. The process is partially planned, but at the same time driven by events and availability of resources (Dahl, Sørby, and Nytrø 2004; Clancy, Effken, and Pesut 2008). The dynamism and complexity of this environment led to the adoption of process oriented approaches and enterprise modeling to the management of organizational operations.

Recently, business process management (BPM) has become to be considered a key valuable asset in the healthcare domain (Kannry 2006). It is increasingly adopted by healthcare organizations because it helps 
improving healthcare processes by taking into account the increasing complexity in patient treatment and the continuous reduction of available resources (Antonacci et al. 2016).Various modeling languages have been developed to cover different aspects of Business processes and organizations. In this context, the Business Process Modeling and Notation (BPMN) standardized by the Object Group Management $(\mathrm{OMG})$ has a pivotal role. BPMN enables modeling problems on an abstract level and facilitate execution and re-usage and supports communication between domain experts and computer scientist (García-Rojo, Blobel, and Laurinavicius 2012). Its adoption in healthcare organizations helps to facilitate the management of complex hospitals; otherwise it can be a good tool for improving the healthcare service quality by providing models in an explicit and understandable way for all participants. Nevertheless the resource management is not integrated and only roles are described in BPMN representation, while at simulation identification and allocation of resources is essential.

In this respect the use of modeling and simulation approach can be considered important for healthcare processes, it helps and supports the decision making process, reduces cost that can occurs in the case wrong decisions haven't been anticipated and as final objective provides a good quality of services. The use of this technique in healthcare management has founded to be promising for the improvement of the outcome and efficiency of care, because it allows to identify and test different potential service designs through quantitative evidence-informed analysis, which take into account quality and safety issues, as well as the cost impact (Kelton 1996). Thus, we want to illustrate the use and application of modeling and simulation for modeling and analyzing healthcare processes in the case of emergency department (ED) by specifying the available resources which perform the process. The remainder of this paper is structured as follows: first a state of art of relevant contributions is presented, and then we justify our motivation. Section 3 provides conceptual models and overview about the methodology. We discuss our modeling and simulation model for ED and report our results in section 4. Finally, conclusions and plans for future work are presented in Section 5.

\section{RELATED WORK}

Modeling and simulation in healthcare sector is thriving. Since data analysis alone cannot give insight into healthcare systems that are rapidly evolving into complex and dynamic systems of system (Gehlot, Matthew, and Sloane 2016). Many researchers argued the effectiveness of Modelling and Simulation in healthcare departments, such as: emergency departments, operating suits, nursing units, ambulatory patient care and ancillary services. Discrete event simulation (DES), system dynamics (SD) and agentbased simulation (ABS) are the main three approaches used when simulating healthcare systems (Jain et al. 2011).

Agent based simulation is being early used in healthcare for studying the disease epidemic, which is considered as one of the main health problem leading to massive death. In order to well analyze the situation mathematical modelling techniques have been qualified as efficient for studying infectious diseases such as flu and others to predict their spreading and anticipate decisions about public health. These models help to identify precaution gaps and predict the outcome of the epidemic. In this case simulation ultimately helps to save lives. Whether ABS is little used in emergency departments compared to DES and SD, it proved a high utility for evaluating workflow and assessing patient diversion policies (Jain et al. 2011). Through system dynamics (SD) applications in healthcare are not as common as DES (S. Brailsford and Harper 2008). They have a long history in the analysis of complex problems. Healthcare organizations began to use SD for the purpose of improving performance in emergency department. In (Royston et al. 1999), the initial aim was to expose the structure of the UK healthcare system by considering the interactions among its different parts. Then, SD has been applied to issues of population health like epidemiology including cervical cancer, diabetes and dengue fever (Homer and Hirsch 2006). 
In DES; entities such as objects that have attributes, experience events, consume resources, and enter queues, over time, have characteristics which determine their pathway through the network as the same way as patients have individual characteristics which determine their pathway through the hospital system (S. C. Brailsford 2007). In the same context, authors in (Norouzzadeh et al. 2015) show how modelling and simulation of internal medicine practice process can help on decision making. The results of simulation, based principally on patient waiting time were able to give an idea about improving resources utilization. Another example is given by Günal and Pidd in (Gehlot, Matthew, and Sloane 2016), which describes a DES model of the process flow of patients, that represents the multitasking behavior of medical staff (doctors and nurses), the only issue is that the ignorance of other possible factors like: doctors' interactions with patients, other medical staff and their working environment lead to incomplete consideration of the problem (Jain et al. 2011).

According to (S. C. Brailsford 2007) healthcare organizations can be hugely complex and therefore require a modelling approach capable of dealing effectively with complexity. The reason behind the use of BPMN is to provide for healthcare management people a standardized description which allows representing the system in a graphical way. The adoption of BPMN as a workflow in healthcare organizations can help facilitate the management of complex hospital process and improve the quality of healthcare as well. It is argued that healthcare processes are highly complex, but the use of BPMN can reduce their complexity by providing a simplified understandable representation of the system. It gives a simplified overview of the behavior of the system and different resources which are contributing on the achievement of the process. Moreover, in BPMN process both human and non-human resources are typically considered available for executing required tasks. Though, one of the main challenges in healthcare organizations is resource management, so to overcome this limitation agent based modeling $(\mathrm{ABM})$ is a suitable paradigm for simulating and modeling healthcare systems and applications where resources, data, control and services are widely distributed. Agent based modeling (ABM) has recently been used for a variety of applications. It offers various types of agents, models of their behavior and characteristics, through a range of architectures and components libraries. It represents objects and populations at an elemental or individualistic level which reflects behaviors of those objects through space and time. These models operate from the bottom up and sometimes generate emergent spatial and temporal patterns at more aggregate levels.

In the present work we propose a new architecture that overcomes some limitations linked to BPMN in healthcare sector. First, we give an overview about the architecture, and then we study its feasibility in the case of emergency department (ED). For this, we propose a BPMN model of a patient pathway in ED which is quite simple but can represent from a high level description realistic case in France. This BPMN model has been created using SLMToolBox editor, which is a software tool developed in the frame of European MSEE project (Bazoun et al. 2016). The SLMToolBox is used by enterprises willing to develop a new service or improve an existing one, within a single enterprise or a virtual manufacturing enterprise. It is used at the stage of "requirement" and "design" of the service engineering process.

Discrete Event system Specification (DEVS) (Zeigler, Praehofer, and Kim 2000) is formalism for modeling discrete events systems. The hierarchical and modular structure of DEVS allows defining multiple models that are coupled to work together in a single and modeling by connecting their input and output through messages (Wainer 2009). In the same way, the resulting model can also be coupled with other models defining multiple layers in the hierarchical structure. In DEVS, atomic models define the behavior of the system, and coupled models describe the structure of the system. There are a large number of DEVS based simulators. Each DEVS simulator has a certain peculiarity that makes it more fit in certain applications. In this study, the proposed DEVS is implemented using VLE (Virtual Laboratory Environment). VLE software (Quesnel, Duboz, and Ramat 2009) implements DEVS M\&S and supports multi-modeling, simulation and analysis. It is based on an extension of DEVS, the Dynamic Structure Discrete Event formalism (DSDE) (Barros 1997). The implementation of the DSDE abstract simulators gives to VLE the ability to simulate distributed models and to load and/or delete atomic and coupled models at runtime. It is also possible to perform statistical analysis of results thanks to a plug-in that 
allows communication between VLE and R (Quesnel, Duboz, and Ramat 2009). In (Bouanan et al. 2016), authors used VLE to instantiate a DEVS model from the description of a social network (represented by individual attributes and relationships). This simulation is used to study the dynamicity of the network (i.e. propagation of information).

On the whole, literature has shown modeling and simulation to be an effective tool used to improve the process and/or outcomes of healthcare systems (Norouzzadeh et al. 2015). It allows identifying and testing different potential service designs through quantitative evidence-informed analysis, which takes into account quality and safety issues, as well as the cost impact (Antonacci et al. 2016). However, the big challenge is still to choose the adequate simulation system according to healthcare indicators to follow.

\section{CONTRIBUTIONS}

According to section 2 we propose a new architecture that overcomes the different limitations regarding modelling and simulation in healthcare organizations.

\subsection{Healthcare Modeling and Simulation architecture}

The proposed architecture has been initiated to be applied to a population subset of the "Nouvelle Aquitaine" region in southwest of France. On the one side, the population has been extracted from an open data source. On the other side, a healthcare resources database, available for French research institutes containing information about healthcare networks has been exploited to spot the selected resources in the chosen region. Interactions between the concerned populations are described through BPMN diagram. In this context and in order to ensure interoperability between existing work in (Bazoun et al. 2016) and our architecture, we propose the reuse of a specific xml file that is generated by SLMToolBox and which provides the component of the BPMN diagram, this file is then used to implement DEVS models thanks to VLE. As recalled previously BPMN describes a process in a static way, so in order to take into account the dynamism of resources we propose to model relation between agents in a dynamic multilayer network structure. For this reason, we propose a mapping between pools in BPMN and layers in network architecture, each layer describes a level of connection between different resources. This method allows us the integration of different relationships between actors in BPMN that are represented with a node in network representation. Actors are defined as agents, which are represented in the agent based model as individuals or group of individuals. Each agent is described by a set of attributes distinguished into two categories:

- Static attributes i.e., gender, and status.

- Dynamic attributes (variables) i.e., availability and state.

Static attributes are intrinsic or unchanged parameters, i.e., time has no effect on them. Dynamic attributes evolve with time or events (Bouanan et al. 2016) (Ruiz-Martin et al. 2016). For example, doctors can be reached or not by their availability depending on working time and number of patient. The DEVS diagram is then used for simulation. Final results would be compared to those estimated by experts in the beginning, with that we aim to improve by our architecture in order that it covers the various existing situations or structures in healthcare organizations thanks to BPMN and DEVS based modelling and simulation. We draw an overview about our architecture in Figure 1; next we will describe the health system in France. 


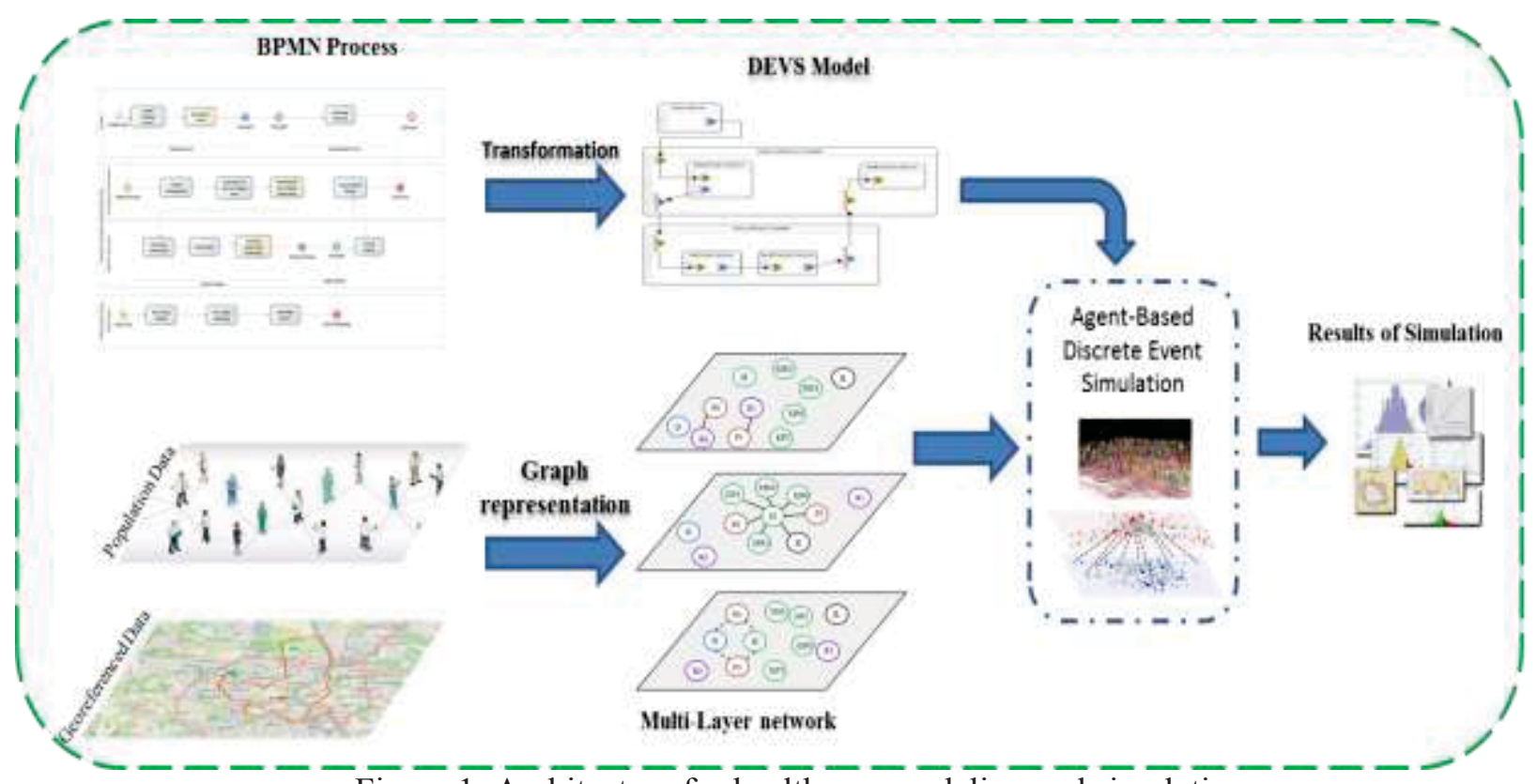

Figure 1: Architecture for healthcare modeling and simulation.

\subsection{Healthcare structure in France}

The French healthcare system is one universal social protection largely financed by government national health insurance. In its 2000 assessment of world health care systems, the World Health Organization found that France provided the "best health care" in the world. Otherwise, the system has two components which are: health care and health insurance. Health care is organized according two sectors: the primary health sector which deals with general health problems and the hospital sector.

Except in the emergency that requires the hospital, French patient usually goes to family doctor, also called "generalist practitioner" or "referring doctor" and who redirects the patient to a specialist, hospital, or an approved health professional. French patients are completely free to use any doctor or hospital they wish. They may also, in some cases, go directly to a specialist either outside or within a hospital. They can choose public or private care by taking into account numerous factors including courtesy, length of appointment, waiting times, cost (in private domain), cleanliness, catering and privacy. Emergency department is the hospital service in charge of welcoming and taking care of the sick and wounded who present themselves or are brought by the emergency services (ambulance...). In some hospitals, there are specialized emergencies (maternity, psychiatry, cardiology...), Patients arrive in emergency departments without an appointment and sometimes at the same time (eg after a road accident), also they have to follow a lot of instructions which makes emergencies a very complex system (Figure 2).

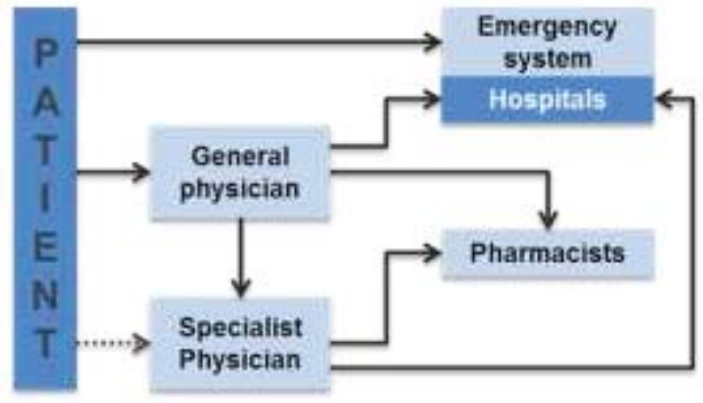

Figure 2: Healthcare organization in France. 


\subsection{Methodology}

As healthcare processes are complex to manage and simulate, we propose here different steps followed for modeling and simulation. The objective at the end is to give the possibility to professional of healthcare to define the model by himself. So we have chosen a category of model that can be used by non-specialist of M\&S.

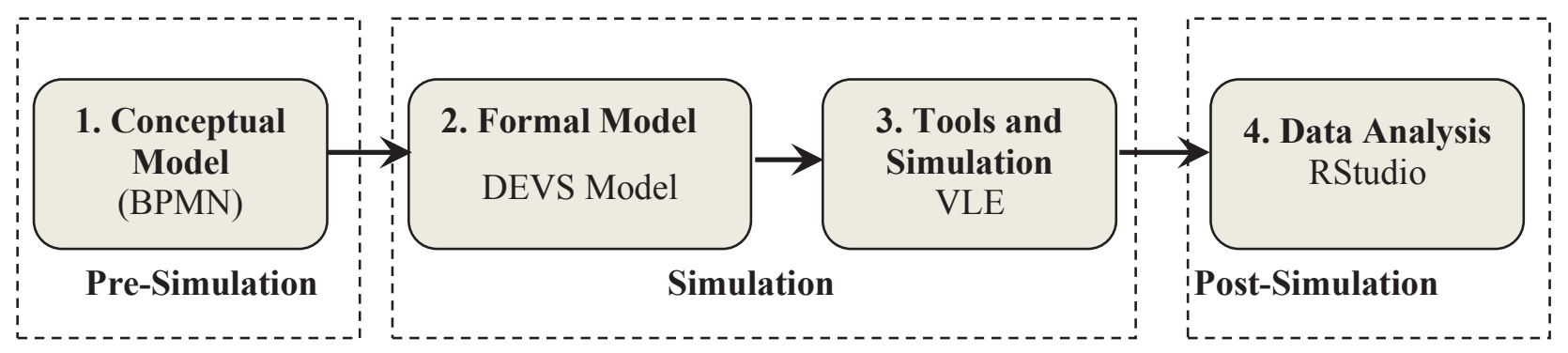

Figure 3: Block diagram for modeling healthcare systems.

The architecture in figure 3 is divided as below:

Pre-Simulation: we define a conceptual model of emergency department (ED) using BPMN. BPMN is created using MSTB, each pool of BPMN describes a participant in the ED workflow.

Simulation: The process starts by transforming the BPMN model of ED to a formal model. Each agent is specified as an atomic model. BPMN model is then specified as a coupled DEVS model. DEVS is used for simulation which is run using VLE.

Post-Simulation: we use $\mathrm{R}$ to process the results. $\mathrm{R}$ is a programming language for statistical computing and graphics. RStudio is used to visualize the simulation results and to conduct analysis.

\section{EMERGENCY DEPARTEMENT DEFINITION AND SIMULATION}

An emergency department (ED) is a clinical unit to treat patients with urgent need of medical care (Smith 1999). It provides care for life-threatening and other emergency cases over 24 hours daily, 7 days a week. Therefore, such departments are highly frequented by patients and this frequency is continuously increasing (Weng et al. 2011). As a result, the performance of emergency departments faces recurrent problems such as overcrowding and congestion. These problems lead to negative effects such as decreased physician productivity, miscommunication between working staff, ambulance diversions (Paul, Reddy, and DeFlitch 2010), and dissatisfaction of patients who may sometimes leave without treatment (Saghafian et al. 2012). Moreover, it leads to high levels of stress, violence, decreased morals among ED staff, increased medical errors, higher mortality rates and high staff turnovers (Kuo, Leung, and Graham 2012). For these reasons, modeling and simulation can be useful to overcome such problems.

\subsection{Emergency Department workflow}

In order to apply the architecture described in the previous section, here we present a case study based on a workflow of patient pathway in ED. In this case, agents are specified in terms of a set of roles they play, in our case we have defined the following roles:

1. Patient (P): Person who seeks for health care.

2. General Practitioner (G): A family doctor, General Physician

3. Emergency Practitioner (EP): Medical Doctor or Nurse who assesses the patient's medical problems, and affects patient to the available doctor. 
4. Medical Specialist (MS): Doctor who is responsible of taking care of patient, providing prescriptions and defining whether a radiology assistance is required or not.

5. Radiologist (R): Physician who adds value to the health care system by generating and interpreting diagnostic images.

Figure 4 illustrates thanks to BPMN model the different steps followed by a patient received at an ED. This figure identifies also the decision tasks (Orange tasks T1 T2 T3 in figure 4) that are connected to the ABM social network model.

The ABM represents the different organizational structures (Geography, Hospital Organizational Structure and Network, etc.).

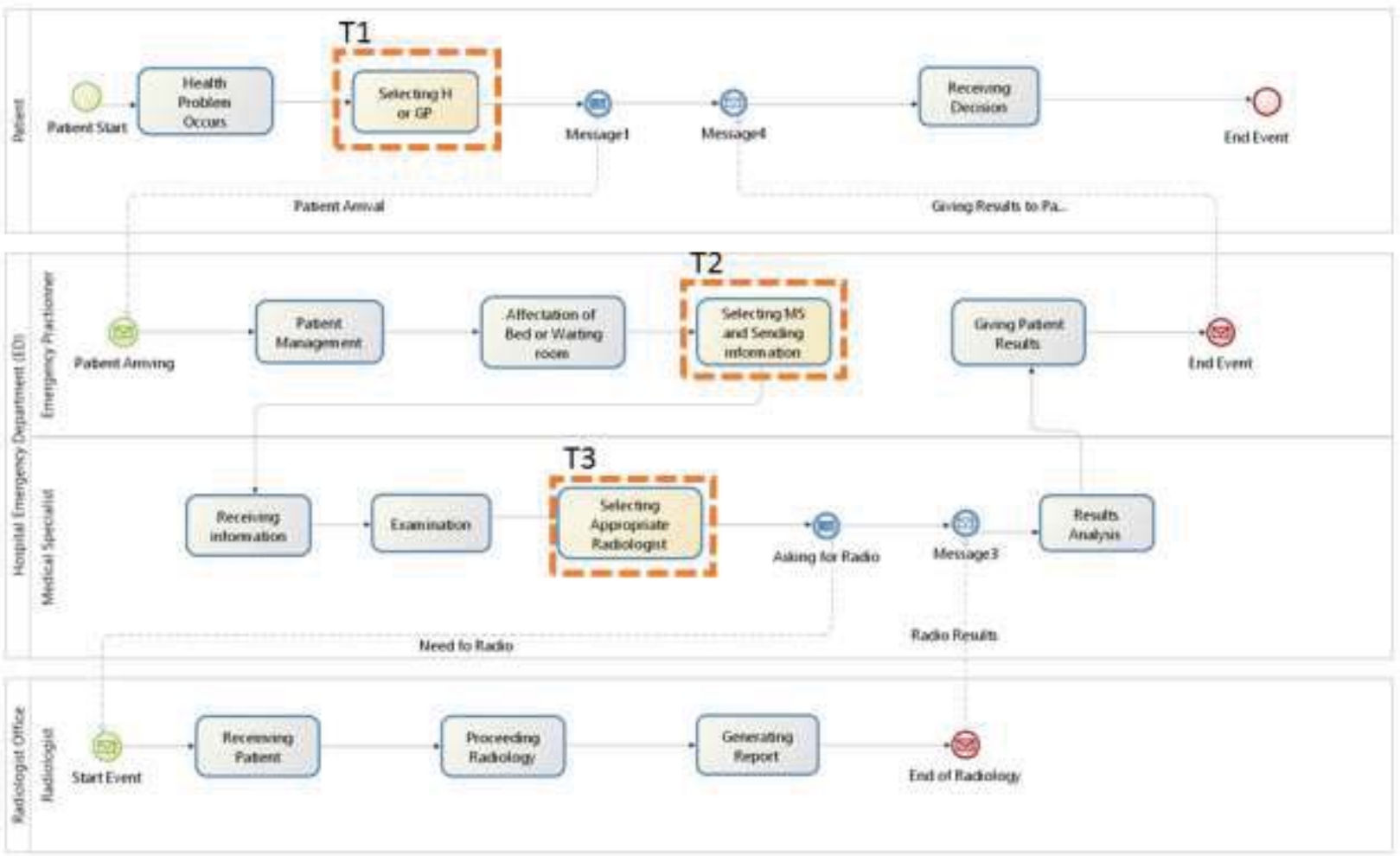

Figure 4: BPMN model for ED Process Workflow.

In an ED, patient's' care may depend only on going to the nearest hospital; it also requires an appropriate allocation of human and material resources in order to increase its effectiveness and efficiency which led to reduce the patient waiting time.

Nevertheless, management and availability of resources is not represented in BPMN as reported in (D'Ambrogio and Zacharewicz 2016). According to that, we propose to model the resource allocation triggered by BPMN diagram as multiplex social networks in which agents interact through multiple interaction channels (layers). For this, the organizational DEVS models network used to implement agent models has used the DEVS network structure defined in (Bouanan et al. 2016) where DEVS network classes are built using VLE.

A representation of a simplified patient resource allocation has been represented as DEVS based layered network as described in Figure 5. In the following, the case of patient (P0) is detailed. We can observe the patient is involved in 3 different networks layers that are graphically represented in grey. 
- Figure 5 (1) represents the network constituted by family doctors and hospitals connected to patients according to distance criteria defined at the setting of the model. We assume that every single patient is connected to one $(\mathrm{G})$ and one or several $(\mathrm{H})$.

- Figure 5 (2) represents the organizational network of the Hospital.

- Figure 5 (3) represent a kind of "yellow page" service, it means that the patient will find specific resources (Medical Specialist and Radiologist) in this network according to their proximity, their reputation (positive opinion of the resource according to social networks information) and the cost (public or private medical services may differ on the additional cost that can exist).

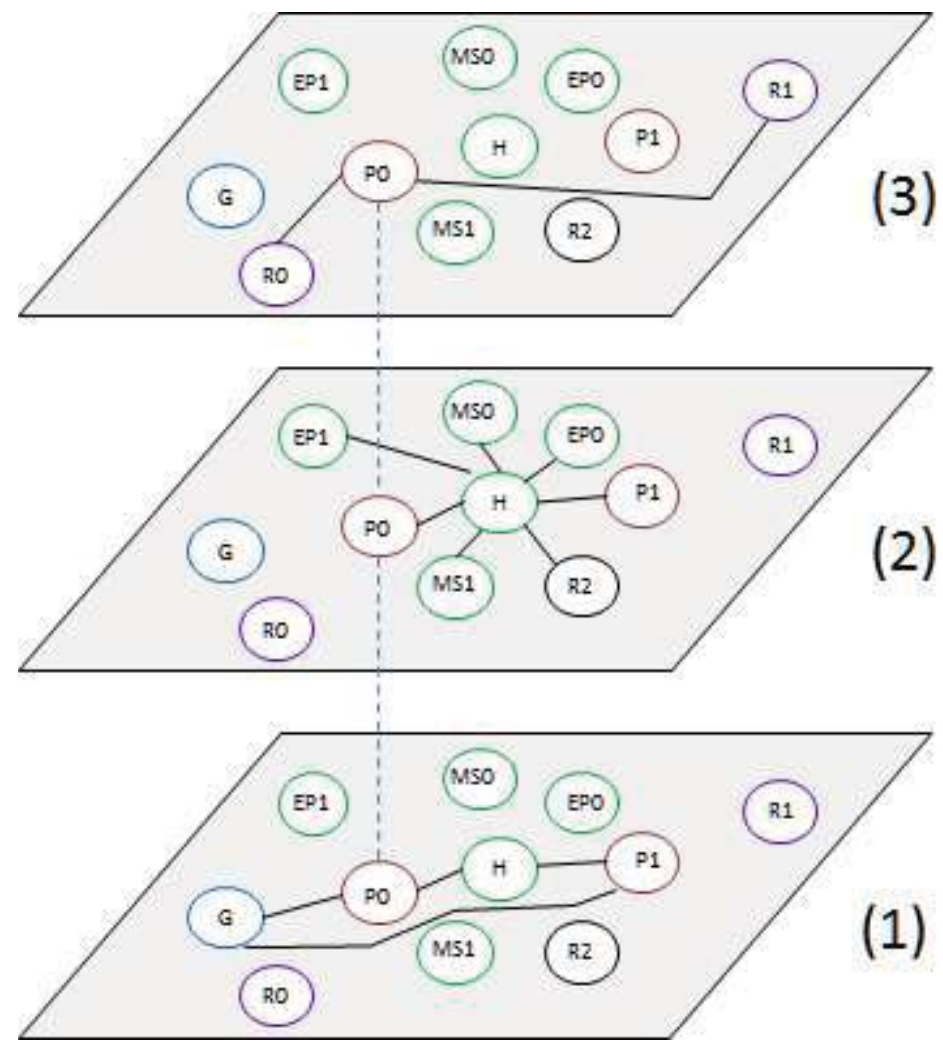

Figure 5: Sketch of the DEVS Model Organizational Architecture.

Overall, once a patient is affected by a health related issue. He launches an internal process (a pool of the BPMN process). He chooses one General Practitioner $(\mathrm{G})$ or one Hospital $(\mathrm{H})$ among the ones that are connected to him in proximity network (1). The choice can be based on the shorter distance or the availability. In the case of emergency department, the patient chooses $\mathrm{H}$ that creates a new connection with patient, emergency practitioners (EP0 and EP1), Medical Specialist (MS0 and MS1) and its internal radiologist $\mathrm{R}$. In that case the search for the appropriate MS and EP is triggered in the ABM network according to their availability, number of patients, working time and the state of patient. After the examination MS may propose a radiology exam to his patient, generally the internal center. In parallel, the patient can search himself other alternatives based on specific criteria. Criteria can be distance from the patient's home, price (with agreement so fully covered or in part covered), reputation etc. the third layer (3) describes the connection between the patient and the chosen radiologist. 


\subsection{Discussion}

In order to study the feasibility of the architecture, we have implemented a simple case coupling the approach proposed by (Bouanan et al. 2016) with BPMN to DEVS (Bazoun et al. 2014). This approach implements a GraphLoader tool that instantiates DS-DEVS models from networks (graphs) by using nodes and links properties. DS-DEVS is used for dynamic structure of DEVS and it preserves all the properties of DEVS (modularity and hierarchical decomposition). Also, we have proposed to study the case of a single patient who is connected in the initial state to all MSs and EPs available in the ED and destroying the connection with the radiologist. We study this simple case at first by taking into account only the availability criteria function.

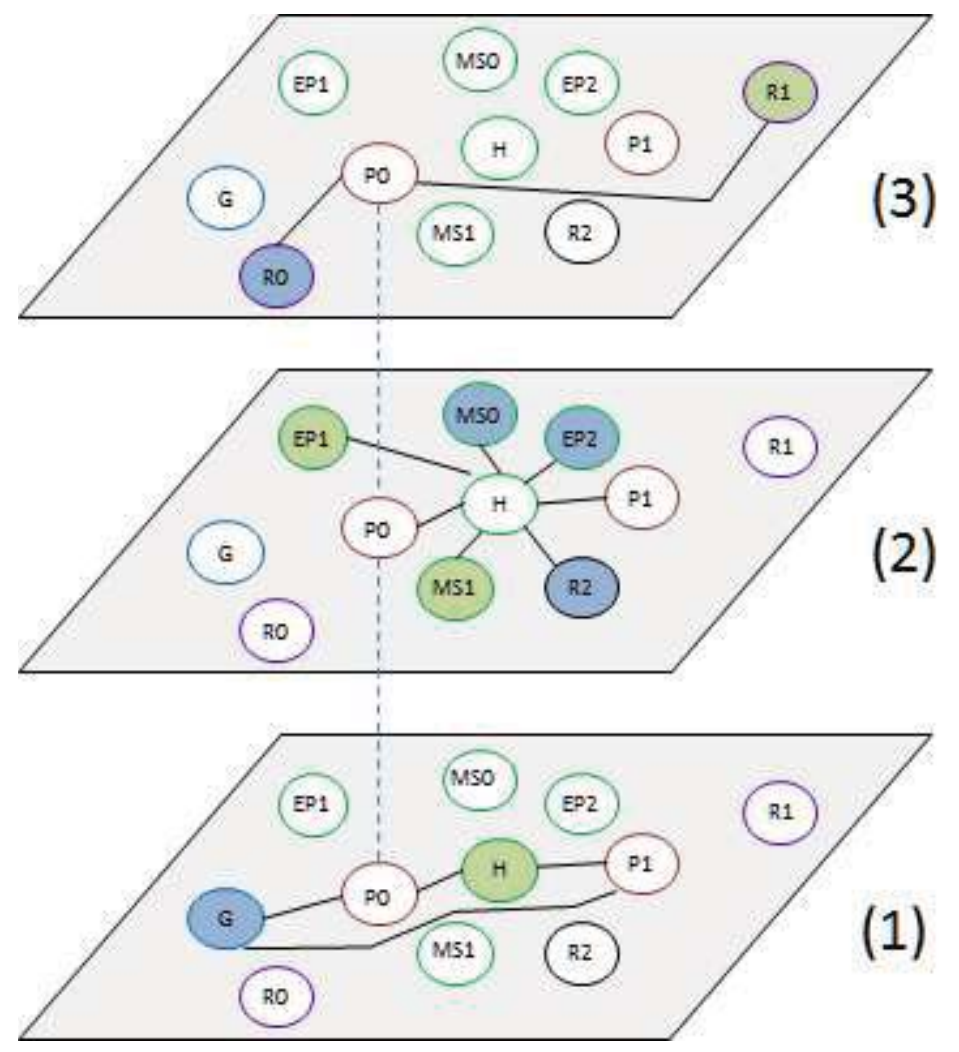

Figure 6: Simulation results of active resources.

Figure 6 (layer 1) shows connections between patient P0, a Family Doctor $(\mathrm{G})$ and the hospital $(\mathrm{H})$, both two last depicted in color in the picture. In the described case, during the first Orange task (T1) of the BPMN model, the patient is selected regarding some emergency criterias the ED of H instead of G. Then, in Figure (layer 2), $\mathrm{H}$ that is connected to a set of resources (colored in layer 2) has solicited an EP and a MS among its connections at the time the BPMN model is reaching the dedicated task (T2). The result shows that MS1 and EP0 have been activated (among MS0 and EP2) and affected to take care of the patient based on their accessibility and availability. Then according to the following of the BPMN process, the search for a radiologist has been triggered in T3. One radiologist has been recommended and directly solicited by H (R2) in Figure 6 (layer 2) and two (R0 and R1) have been solicited directly by the patient in Figure 6 (layer 2). According to multi criterias evaluation (including distance from patient home, availability, cost and positive opinion), the radiologist R1 has been chosen by the patient.

At the end, in this illustration, the simulation has permitted to identify and select resources to support the execution of the BPMN model. In detail, green agents represent all resources that have been serving patient P0 while blue agents that represent all solicited but unavailable or not selected resources for P0. 


\section{CONCLUSIONS}

This paper presents a new architecture based on business process and multidimensional network for modeling in healthcare sector. It uses BPMN to define the patient pathway process connected with an $\mathrm{ABM}$ network. It defines and run the search for resources and their allocation based on a multi-layer different healthcare stakeholders networks exploration. The objective is to study the impact of dynamic allocation of participant actors in BPMN healthcare pathways.

The proposed architecture has been evaluated for an Emergency Department in this paper, whose organizational complexity and dynamic nature can make them difficult to characterize. In the study case we have taken into consideration a reduced set of criterias to select the resources including: proximity from patient, availability, random given price and opinion. These criterias have been used to evaluate different solutions such balancing resources recommended by the hospital regarding patient own choice for instance.

As future work, the proposed solution will progressively integrate more real data from ED effective practices, as well as more demographic information of patients and staff, budget constraints. The BPMN pathway model will be confronted to specialist to become more robust, such as integrating possible situations and problems that can face the patient. The final goal will consist in giving the user the control on the BPMN model and on the resource networks description. It will permit observing thanks to simulation a territory regarding its capacity to handle population health issues. This question is crucial especially in the context of low population density zone and rural medicine (deserts in terms of medical care are a preoccupation in Europe these days).

\section{ACKNOWLEDGMENTS}

We thank Pr. Bruno Vallespir for his assistance in data acquisition for the healthcare structure.

\section{REFERENCES}

Antonacci, G., A. Calabrese, A. D’Ambrogio, A. Giglio, B. Intrigila, and N. Levialdi Ghiron. 2016. "A BPMN-Based Automated Approach for the Analysis of Healthcare Processes." In Enabling Technologies: Infrastructure for Collaborative Enterprises (WETICE), 2016 IEEE 25th International Conference on, 124-129.

Barros, Fernando J. 1997. "Modeling Formalisms for Dynamic Structure Systems." ACM Trans. Model. Comput. Simul. 7 (4): 501-515.

Bazoun, Hassan, Gregory Zacharewicz, Yves Ducq, and Hadrien Boye. 2016. "SLMToolBox: enterprise service process modelling and simulation by coupling DEVS and services workflow" In Int. J. Simulation and Process Modelling, Vol. 11, No. 6, 2016

Brailsford, Sally C. 2007. "Advances and Challenges in Healthcare Simulation Modeling: Tutorial.” In Proceedings of the 39th Conference on Winter Simulation: 40 Years! The Best Is yet to Come, 14361448.

Brailsford, Sally, and Paul Harper. 2008. “OR in Health.” European Journal of Operational Research 185

Bouanan, Youssef, Gregory Zacharewicz, Bruno Vallespir, Judicael Ribault, and Saikou Y. Diallo. 2016. "DEVS Based Network: Modeling and Simulation of Propagation Processes in a Multi-Layers Network." In Proceedings of the Modeling and Simulation of Complexity in Intelligent, Adaptive and Autonomous Systems 2016 (MSCIAAS 2016) and Space Simulation for Planetary Space Exploration (SPACE 2016), 8:1-8:8. MSCIAAS '16. San Diego, CA, USA: Society for Computer Simulation International.

Clancy, Thomas R., Judith A. Effken, and Daniel Pesut. 2008. “Applications of Complex Systems Theory in Nursing Education, Research, and Practice." Nursing Outlook 56 (5): 248-256. 
Dahl, Yngve, Inger Dybdahl Sørby, and Øystein Nytrø. 2004. "Context in Care-Requirements for Mobile Context-Aware Patient Charts." In Proceedings of the 11th World Congress on Medical Informatics (MEDINFO). IOS, Amsterdam, 597-601.

D'Ambrogio Andrea and Gregory Zacharewicz. 2016. "Resource-based modeling and simulation of business processes". In Proceedings of the Summer Computer Simulation Conference (SCSC '16). Society for Computer Simulation International, San Diego, CA, USA, , Article 63, 8 pages.

García-Rojo, Marcial, Bernd Blobel, and Arvydas Laurinavicius. 2012. Perspectives on Digital Pathology: Results of the COST Action IC0604 EURO-TELEPATH. IOS Press.

Gehlot, Vijay, Liji Matthew, and Elliot B. Sloane. 2016. "From Data to Processes - Use of Modeling and Simulation in Healthcare." In 2016 IEEE-EMBS International Conference on Biomedical and Health Informatics (BHI), 320-323.

Homer, Jack B., and Gary B. Hirsch. 2006. "System Dynamics Modeling for Public Health: Background and Opportunities." American Journal of Public Health 96 (3): 452-458.

Quesnel, Gauthier, Raphaël Duboz, and Éric Ramat. 2009. "The Virtual Laboratory Environment-An Operational Framework for Multi-Modelling, Simulation and Analysis of Complex Dynamical Systems." Simulation Modelling Practice and Theory 17 (4): 641-653.

Jain, S., R. R. Creasey, J. Himmelspach, K. P. White, M. Fu, Paula Escudero-marin, and Michael Pidd. 2011. "USING ABMS TO SIMULATE EMERGENCY DEPARTMENTS."

Kannry, Joseph. 2006. "Use of Simulation Approaches in the Study of Clinician Workflow."

Kelton, W. David. 1996. "Statistical Issues in Simulation." In Proceedings of the 28th Conference on Winter Simulation, 47-54.

Kuo, Yong-Hong, Janny MY Leung, and Colin A. Graham. 2012. "Simulation with Data Scarcity: Developing a Simulation Model of a Hospital Emergency Department." In Proceedings of the 2012 Winter Simulation Conference (WSC), 1-12.

Norouzzadeh, Shaghayegh, Nancy Riebling, Lawrence Carter, Joseph Conigliaro, and Martin E. Doerfler. 2015. "Simulation Modeling to Optimize Healthcare Delivery in an Outpatient Clinic." In 2015 Winter Simulation Conference (WSC), 1355-1366.

Paul, Sharoda A., Madhu C. Reddy, and Christopher J. DeFlitch. 2010. "A Systematic Review of Simulation Studies Investigating Emergency Department Overcrowding." Simulation 86 (8-9): 559571.

Quesnel, Gauthier, Raphaël Duboz, and Éric Ramat. 2009. "The Virtual Laboratory Environment-An Operational Framework for Multi-Modelling, Simulation and Analysis of Complex Dynamical Systems." Simulation Modelling Practice and Theory 17 (4): 641-653.

Royston, Geoff, Ayesha Dost, Jeremy Townshend, and Howard Turner. 1999. "Using System Dynamics to Help Develop and Implement Policies and Programmes in Health Care in England." System Dynamics Review 15 (3): 293.

Ruiz-Martin, Cristina, Gabriel Wainer, Youssef Bouanan, Gregory Zacharewicz, and Adolfo López Paredes. 2016. "A Hybrid Approach to Study Communication in Emergency Plans." In Winter Simulation Conference (WSC), 2016, 1376-1387.

Saghafian, Soroush, Wallace J. Hopp, Mark P. Van Oyen, Jeffrey S. Desmond, and Steven L. Kronick. 2012. "Patient Streaming as a Mechanism for Improving Responsiveness in Emergency Departments." Operations Research 60 (5): 1080-1097.

Wainer, Gabriel A. 2009. Discrete-Event Modeling and Simulation: A Practitioner's Approach. CRC Press. 
Weng, Shao-Jen, Bing-Chuin Cheng, Shu Ting Kwong, Lee-Min Wang, and Chun-Yueh Chang. 2011. "Simulation Optimization for Emergency Department Resources Allocation." In Proceedings of the 2011 Winter Simulation Conference (WSC), 1231-1238.

Zeigler, Bernard P., Herbert Praehofer, and Tag Gon Kim. 2000. Theory of Modeling and Simulation: Integrating Discrete Event and Continuous Complex Dynamic Systems.

\section{AUTHOR BIOGRAPHIES}

MARIEM SBAYOU is a Ph.D. Student at IMS laboratory of University of Bordeaux. Her research domain includes DEVS, Healthcare M\&S. She received her Engineer degree as software engineer from the International Academy of Civil Aviation in Morocco. Her email address is mariem.sbayou@ubordeaux.fr.

YOUSSEF BOUANAN is a Postdoctoral researcher at University of Bordeaux. He received his Ph.D. degree in Production Engineering from University of Bordeaux, France. His research interests include modeling and simulation theory, social network and workflow. His email address is youssef.bouanan@ubordeaux.fr.

GREGORY ZACHAREWICZ is Associate Professor HDR at University of Bordeaux and IMS Lab with both competences in enterprise engineering and M\&S. His email address is gregory.zacharewicz@ubordeaux.fr.

JUDICAEL RIBAULT is a Ph.D. freelance software architect and associate researcher at University of Bordeaux and IMS Lab. His research interests include modeling and simulation. His email address is jribault@gmail.com.

JULIEN FRANCOIS is Associate Professor at University of Bordeaux and IMS Lab His research interests include supply chain, modeling and simulation in industrial sector. His email address is julien.francois@u-bordeaux.fr. 